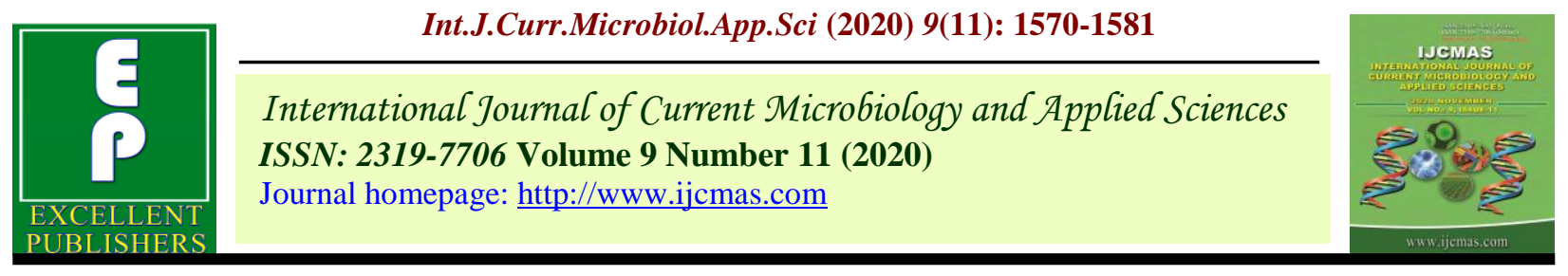

Original Research Article

https://doi.org/10.20546/ijcmas.2020.911.186

\title{
Correlation and Path Coefficient Analysis in Wheat (Triticum aestivum L. em. Thell)
}

\author{
K. P. Singh*, Vinod Singh, Gaurav Sharma, M. P. Chauhan, \\ Tejasvi Singh and R. D. S. Yadav
}

Department of Genetics and Plant Breeding, Acharya Narendra Deva University of Agriculture and technology, Kumarganj, Ayodhya, Uttar Pradesh, India

*Corresponding author

\section{A B S T R A C T}

Keywords

Wheat

(Triticum aestivum),

Correlation and

Path coefficient

Article Info

Accepted:

12 October 2020

Available Online:

10 November 2020
The present study was carried out during Rabi seasons of 2017-18 \& 2018-19 at Main Experimental Station, Acharya Narendra Deva University of Agriculture and Technology, Kumarganj, Ayodhya (U.P.). The experimental materials comprised of twenty genetically diverse varieties/strains and their 105 crosses. The 26 parents were involved in a crossing programme to develop a line $\mathrm{x}$ tester set $(21$ lines +5 testers +3 checks) during Rabi season of 2017-18in Randomized Block Design. The experimental materials were evaluated in two conditions i.e. timely sown $\left(E_{1}\right)$ and late sown $\left(E_{2}\right)$ condition for twelve quantitative characters. The harvest index (0.843), tiller per plant (0.154), spike length (0.146) showed highly positive phenotypic correlation with in $\mathrm{E}_{1}$ for grain yield per plant, whail grain yield per plant exhibited highly positive phenotypic correlation with harvest index $(0.687)$, test weight $(0.111)$ and spikelet per spike $(0.101)$ in $\mathrm{E}_{2}$. The highest positive direct effect on grain yield per plant was exerted vai harvest index (1.340), plant height (0.005), penduncle length (0.003). Whereas $\mathrm{E}_{2}$ showed direct positive effect on grain yield per plant by harvest index (1.624), biological yield (1.179), and flag leaf area (0.016). The highest positive indirect effect on grain yield was exerted by days to maturity $(0.141)$ via biological yield per plant followed by days to $50 \%$ flowering (0.092) via biological yield per plant, test weight (0.040) via biological yield per plant, tiller per plant and spike length (0.005) via biological yield per plant and flag leaf area (0.003) via biological yield per plant in $E_{1}$. While in $E_{2}$ highest positive indirect effect on grain yield showed by plant height $(0.220)$ via biological yield per plant followed by days to maturity $(0.151)$, tiller per plant $(0.053)$ peduncle length $(0.044)$, test weight $(0.033)$.

\section{Introduction}

Wheat, (Triticum aestivum L. em. Thell) the world's largest cereal crop which belongs to Graminae (Poaceae) family of the genus Triticum. It has been described as the 'King of cereals' because of the acreage it occupies, high productivity and the prominent position in the international food grain trade. Wheat is consumed in a variety of ways such as bread chapatti, porridge, flour, suji etc.

The term "Wheat" is derived from many different locations, specifically from English, 
German and Welsh language. Wheat is most commonly defined by all cultures as "that which is white" due to its physical characteristics of light colored crops.

In India, during 2019-20 Rabi season, wheat has been cultivated in 30.55 million hectares constituting 24.34 per cent of the total crop acreage. Indian wheat production in 2019-20 has made another landmark achievement by producing $107.18 \mathrm{mt}$ with an average national productivity of $3508 \mathrm{~kg} / \mathrm{ha}$. During the past year production was also more than 100 million tonnes (103.60 million tonnes) and the current year production has witnessed a change of 3.58 million tonnes $(+3.46 \%)$. The positive growth in production is attributed to the increased area by 4.21 per cent despite a fall in the crop yield marginally by -0.72 percent. Increase in the support price by ${ }^{`} 85$ per quintal in comparison to the recent past year and announced as ' 1925 per quintal of wheat, might have had a positive impact on the crop acreage (+1.24 million hectares). The crop area and productivity have increased in a majority of the states is a main reason behind the landmark production. States like Madhya Pradesh, Maharashtra, Gujarat and Rajasthan have shown a significant increase in the crop area over the past year have resulted in a major quantum jump in overall wheat production Anonymous (2019).

Among the wheat producing states, Uttar Pradesh registered a significant level of crop output estimated at 32.09 million tonnes (30\%), followed by Madhya Pradesh (18.58 million tonnes: 17\%), Punjab (18.21 million tonnes: $17 \%$ ), Haryana (12.07 million tonnes: 11\%), Rajasthan (10.57 million tonnes: 10\%) and Bihar (6.55 million tonnes: 6\%). The aforementioned six states hold a share of about 92 per cent in total wheat production. With the exception of Chhatisgarh, Haryana, Odisha, Punjab, Telangana and Uttar Pradesh, the rest of the states registered an increase in production during 2019-20 relative to 201819. Overall production from all these states has declined by 1.23 million tonnes owing to the fall in yield levels and/or acreage. The highest fall was noticed in Uttar Pradesh (0.65 million tonnes: $-1.99 \%$ ). The increase in wheat production was maximum in the case of Madhya Pradesh (+2.06 million tonnes: $+12.48 \%)$, followed by Gujarat $(+0.85$ million tonnes: $+35.46 \%)$ and Maharashtra (+0.83 million tonnes: $66.20 \%$ ). In percentage terms, it was highest for West Bengal (72.53\%: 0.25 million tonnes), Anonymous (2019)

\section{Materials and Methods}

The present investigation entitled "Studies on combining ability and heterosis for yield and its components under sodic soil in bread wheat (Triticum aestivum L. em. Thell.)" was conducted in RBD in three replications at Main Experiment Station of AcharyaNarendra Deva University of Agriculture and Technology, Kumarganj, Ayodhya (U.P.) during Rabi, 2017-19.

Geographically, experimental site is located between $24^{\circ} 47^{\prime}$ and $26^{\circ} 56^{\prime} \mathrm{N}$ latitude, $82^{\circ}$ $12^{\prime}$ and $83^{\circ} 98^{\prime} \mathrm{E}$ longitude and at an altitude of $113 \mathrm{~m}$ above mean sea level. This area falls in sub-tropical climatic zone. The soil type is sandy loam. The annual rainfall is about 1270 $\mathrm{mm}$. The climate of district Ayodhya is semiarid with hot summer and cold winter.

The experimental materials of the study comprised of 134 treatments of wheat. These materials included $105 \mathrm{~F}_{1}$ 's, 26 parental lines (21 females +5 males) and three standard varieties. Twelve lines were crossed with 5 testers following Line $\mathrm{x}$ Tester mating design during rabi season 2017-18 at Main Experiment Station (MES), AcharyaNarendra Deva University of Agriculture and Technology, Kumarganj, Ayodhya (U.P.). 
Observations on the twelve characters were recorded on 5 randomly selected plants in each replication for following characters viz. days to $50 \%$ flowering, days to maturity, plant height $(\mathrm{cm})$, tillers per plant, spike length $(\mathrm{cm})$, flag leaf area $\left(\mathrm{cm}^{2}\right)$, peduncle length (cm), 1000-grain weight (g), biological yield per plant $(\mathrm{g})$, grain yield per plant $(\mathrm{g})$, harvest index (\%)and spikelets/spike (no.) except days to $50 \%$ flowering and days to maturity which is on plot basis.

\section{Material used in experiment}

Twenty one lines NW-1067, NW-4018, NW1012, PBW-17, PBW-154, PBW-373, PBW343, DBW-39, DBW-14, DBW-22, HD-2733, HD-2824, KRL-392, KRL-20, KRL-391, KRL-393, KRL-99, KRL-213, HI-1563, GW366 and HUW-234) with 5 testers (KRL-1-4, KRL-19, KRL-3-4, NW-2036 and HD-2967 with five tester KRL-1-4, KRL-19, KRL-3-4, NW-2036 and HD-2967 and three check namely, UP-2338, PBW-550 and NW-5054.

\section{Data analysis}

The data thus recorded were subjected to statistical and biometrical analysis as detailed as follows: Correlation coefficient analysis as calculated by Al-Jibouri et al., (1958) to test the significant correlation between the traits. Path coefficient analysis was performed to assess direct and indirect effect of the measured traits on grain yield according to the technique outlined by Dewey and Lu (1959).

\section{Results and Discussion}

The genetic architecture of grain yield in as well as other crops is based on the balance or overall net effect produced by various yield components directly or indirectly by interacting with one another. Therefore, selection for yield per se alone would not matter much as such unless accompanied by the selection for various component characters responsible for conditioning Thus, identification of important components and information about their association with yield and with each other are very useful for developing efficient breeding strategy for evolving high yielding varieties/hybrids. The correlation coefficient is the measure of degree of symmetrical association between two variables or characters which helps us in understanding the nature and magnitude of association among yield and yield components. In the present investigation, phenotypic and genotypic correlation coefficients were computed among 12 characters (Table 1 and 2) in timely $\left(\mathrm{E}_{1}\right)$ and late $\left(\mathrm{E}_{2}\right)$ conditions.

\section{Path coefficient analysis}

Path coefficient analysis is a tool to partition the observed correlation coefficient into direct and indirect effects of yield components on seed yield. Path analysis provides clearer picture of character associations for formulating efficient Selection strategy. The path coefficient analysis using genotypic as well as phenotypic correlation coefficient estimated in $\mathrm{E}_{1}$ and $\mathrm{E}_{2}$ conditions were carried out to asses direct and indirect effects of twelve characters on the expression of grain yield per plant.

The highest positive direct effect on grain yield per plant were exerted by harvest index (1.340), plant height (0.005), penduncle length (0.003) and negative direct effect on grain yield per plant were exerted by test weight (-0.005) and spike length (-0.004) in $\mathrm{E}_{1}$; whereas $\mathrm{E}_{2}$ showed direct effect on grain yield per plant by harvest index (1.624), biological yield (1.179), and flag leaf area (0.016). While negative by tiller per plant ($0.020)$, days to $50 \%$ lowering $(-0.016)$ and plant height (-0.014) in $\mathrm{E}_{2}$. 
Table.1 Estimation of direct and indirect effect of 12 characters on grain yield/ plant at phenotypic and genotypic level under timely sown $\left(\mathrm{E}_{1}\right)$ condition in wheat

\begin{tabular}{|c|c|c|c|c|c|c|c|c|c|c|c|c|c|}
\hline & & $\begin{array}{c}\text { Day to } \\
50 \% \\
\text { flowering }\end{array}$ & $\begin{array}{c}\text { Flag leaf } \\
\text { area } \\
(\mathrm{cm})^{2}\end{array}$ & $\begin{array}{l}\text { Days to } \\
\text { maturity }\end{array}$ & $\begin{array}{c}\text { Plant } \\
\text { height } \\
(\mathrm{cm})\end{array}$ & $\begin{array}{c}\text { Tillers/ } \\
\text { plant }\end{array}$ & $\begin{array}{l}\text { Spike } \\
\text { length } \\
(\mathrm{cm})\end{array}$ & $\begin{array}{l}\text { Peduncl } \\
\text { e length } \\
(\mathrm{cm})\end{array}$ & $\begin{array}{c}\text { Biological } \\
\text { yield/ } \\
\text { Plant } \\
(\mathrm{gm})\end{array}$ & $\begin{array}{c}\text { Test } \\
\text { weight } \\
\text { (gm) }\end{array}$ & $\begin{array}{c}\text { Harvest } \\
\text { index }(\%)\end{array}$ & $\begin{array}{l}\text { Spikelets/ } \\
\text { spike }\end{array}$ & $\begin{array}{l}\text { Grain yield/ } \\
\text { plant (g) }\end{array}$ \\
\hline \multirow[t]{2}{*}{ Days to $50 \%$ flowering } & $\mathrm{P}$ & 0.00190 & 0.00040 & 0.00020 & 0.00000 & 0.00000 & 0.00030 & 0.00030 & 0.09280 & 0.00010 & -0.24490 & -0.00010 & $-0.149 * *$ \\
\hline & $\mathrm{G}$ & 0.016 & -0.001 & 0.001 & 0.000 & -0.007 & 0.000 & -0.002 & 0.158 & 0.000 & -0.376 & -0.001 & $-0.213 * *$ \\
\hline \multirow[t]{2}{*}{ Flag leaf area $\left(\mathrm{cm}^{2}\right)$} & $\mathrm{P}$ & 0.00030 & 0.00250 & 0.00000 & -0.00030 & 0.00000 & -0.00010 & -0.00020 & 0.00310 & 0.00060 & -0.06260 & -0.00010 & -0.057 \\
\hline & G & 0.005 & -0.004 & 0.000 & -0.001 & 0.002 & 0.000 & 0.001 & -0.008 & -0.001 & -0.034 & 0.000 & -0.040 \\
\hline \multirow[t]{2}{*}{ Days to maturity } & $\mathrm{P}$ & 0.00080 & 0.00000 & 0.00050 & -0.00020 & 0.00000 & 0.00110 & 0.00070 & 0.14170 & -0.00100 & -0.36270 & 0.00010 & $-0.219 * *$ \\
\hline & G & 0.007 & 0.000 & 0.002 & 0.000 & -0.009 & 0.001 & -0.006 & 0.258 & 0.001 & -0.622 & 0.001 & $-0.367 * *$ \\
\hline \multirow[t]{2}{*}{ Plant height (cm) } & $\mathrm{P}$ & 0.00000 & -0.00010 & 0.00000 & 0.00590 & 0.00000 & 0.00010 & 0.00030 & -0.03980 & -0.00060 & 0.09560 & -0.00010 & 0.061 \\
\hline & $\mathrm{G}$ & 0.000 & 0.000 & 0.000 & 0.007 & -0.001 & 0.000 & -0.002 & -0.064 & 0.001 & 0.141 & 0.000 & 0.083 \\
\hline \multirow[t]{2}{*}{ Tillers/plant } & $\mathrm{P}$ & -0.00010 & 0.00010 & 0.00000 & -0.00020 & 0.00050 & -0.00010 & 0.00030 & 0.00580 & 0.00080 & 0.14690 & 0.00010 & $0.154 * *$ \\
\hline & G & -0.003 & 0.000 & -0.001 & 0.000 & 0.034 & 0.000 & -0.006 & -0.045 & -0.002 & 0.488 & 0.001 & $0.465^{* *}$ \\
\hline \multirow[t]{2}{*}{ Spike length (cm) } & $\mathrm{P}$ & -0.00010 & 0.00010 & -0.00010 & -0.00020 & 0.00000 & -0.00470 & -0.00020 & 0.00580 & 0.00080 & 0.14420 & 0.00000 & $0.146^{* *}$ \\
\hline & $\mathrm{G}$ & -0.001 & 0.000 & -0.001 & 0.000 & 0.001 & -0.003 & 0.001 & 0.033 & -0.001 & 0.211 & 0.000 & $0.239 * *$ \\
\hline \multirow[t]{2}{*}{ Peduncle length (cm) } & $\mathrm{P}$ & 0.00010 & -0.00010 & 0.00010 & 0.00040 & 0.00000 & 0.00020 & 0.00390 & -0.05410 & -0.00090 & 0.03300 & 0.00070 & -0.017 \\
\hline & $\mathrm{G}$ & 0.002 & 0.000 & 0.001 & 0.001 & 0.013 & 0.000 & -0.017 & -0.074 & 0.001 & 0.043 & 0.005 & -0.025 \\
\hline \multirow[t]{2}{*}{ Biological yield/plant (g) } & $\mathrm{P}$ & 0.00020 & 0.00000 & 0.00010 & -0.00030 & 0.00000 & 0.00000 & -0.00030 & 0.73110 & -0.00030 & -0.91360 & -0.00010 & $-0.183 * *$ \\
\hline & G & 0.003 & 0.000 & 0.001 & -0.001 & -0.002 & 0.000 & 0.002 & 0.741 & 0.000 & -1.007 & -0.001 & $-0.264 * *$ \\
\hline \multirow[t]{2}{*}{ Test weight (g) } & $\mathrm{P}$ & 0.00000 & -0.00030 & 0.00010 & 0.00070 & -0.00010 & 0.00070 & 0.00070 & 0.04090 & -0.00530 & -0.27320 & 0.00030 & $-0.236^{* *}$ \\
\hline & $\mathrm{G}$ & 0.000 & 0.001 & 0.001 & 0.001 & -0.013 & 0.001 & -0.003 & 0.049 & 0.005 & -0.321 & 0.002 & $-0.279 * *$ \\
\hline \multirow[t]{2}{*}{ Harvest index (\%) } & $\mathrm{P}$ & -0.00040 & -0.00010 & -0.00010 & 0.00040 & 0.00000 & -0.00050 & 0.00010 & -0.49810 & 0.00110 & 1.34090 & -0.00010 & $0.843^{* *}$ \\
\hline & G & -0.004 & 0.000 & -0.001 & 0.001 & 0.012 & -0.001 & -0.001 & -0.538 & -0.001 & 1.387 & -0.001 & $0.854 * *$ \\
\hline \multirow[t]{2}{*}{ Spikelets/Spike } & $\mathrm{P}$ & -0.00010 & -0.00010 & 0.00000 & -0.00010 & 0.00000 & 0.00000 & 0.00100 & -0.01850 & -0.00060 & -0.06890 & 0.00280 & -0.084 \\
\hline & $\mathrm{G}$ & -0.001 & 0.000 & 0.000 & 0.000 & 0.002 & 0.000 & -0.008 & -0.047 & 0.001 & -0.076 & 0.011 & $-0.116^{*}$ \\
\hline
\end{tabular}

*, ** significant at 5 and 1 per cent probability levels, respectively 
Table.2 Estimates of direct and indirect effect of 12 characters on grain yield per plant at phenotypic and genotypic level under late sown $\left(\mathrm{E}_{2}\right)$ condition in wheat

\begin{tabular}{|c|c|c|c|c|c|c|c|c|c|c|c|c|c|}
\hline & & $\begin{array}{c}\text { Days to } \\
50 \% \\
\text { flowering }\end{array}$ & $\begin{array}{l}\text { Flag } \\
\text { leaf } \\
\text { area } \\
\left(\mathrm{cm}^{2}\right)\end{array}$ & $\begin{array}{l}\text { Days to } \\
\text { maturity }\end{array}$ & $\begin{array}{c}\text { Plant } \\
\text { height } \\
(\mathrm{cm})\end{array}$ & $\begin{array}{c}\text { Tillers/ } \\
\text { plant }\end{array}$ & $\begin{array}{l}\text { Spike } \\
\text { length } \\
(\mathrm{cm})\end{array}$ & $\begin{array}{l}\text { Peduncle } \\
\text { length }(\mathrm{cm})\end{array}$ & $\begin{array}{l}\text { Biological } \\
\text { yield/ plant } \\
\text { (g) }\end{array}$ & $\begin{array}{c}\text { Test } \\
\text { weight } \\
\text { (g) }\end{array}$ & $\begin{array}{c}\text { Harvest } \\
\text { index }(\%)\end{array}$ & Spikelets/spike & $\begin{array}{c}\text { Grain } \\
\text { yield/ } \\
\text { plant (g) }\end{array}$ \\
\hline \multirow[t]{2}{*}{ Days to $50 \%$ flowering } & $P$ & -0.0162 & -0.0018 & 0.0000 & -0.0019 & -0.0004 & -0.0003 & -0.0004 & -0.2042 & 0.0002 & 0.3062 & -0.0005 & 0.081 \\
\hline & $\mathrm{G}$ & -0.134 & 0.048 & 0.022 & 0.028 & 0.014 & 0.028 & -0.079 & -0.594 & -0.003 & 1.008 & -0.094 & $0.244^{* *}$ \\
\hline \multirow[t]{2}{*}{ Flag leaf area $\left(\mathrm{cm}^{2}\right)$} & $\mathrm{P}$ & 0.0018 & 0.0164 & 0.0000 & 0.0005 & 0.0012 & 0.0001 & 0.0001 & -0.1002 & 0.0002 & 0.1463 & 0.0004 & 0.067 \\
\hline & $\mathrm{G}$ & 0.040 & -0.158 & 0.018 & -0.023 & -0.052 & -0.001 & 0.124 & -0.324 & 0.030 & 0.458 & 0.006 & $0.117 *$ \\
\hline \multirow[t]{2}{*}{ Days to maturity } & $\mathrm{P}$ & 0.0011 & 0.0002 & 0.0005 & -0.0007 & -0.0008 & -0.0003 & 0.0000 & 0.1512 & 0.0002 & -0.2273 & -0.0006 & -0.076 \\
\hline & $\mathrm{G}$ & 0.020 & 0.019 & -0.149 & 0.054 & 0.036 & 0.007 & 0.045 & 0.413 & -0.001 & -0.673 & 0.065 & $-0.163 * *$ \\
\hline \multirow[t]{2}{*}{ Plant height (cm) } & $\mathrm{P}$ & -0.0021 & -0.0006 & 0.0000 & -0.0149 & 0.0005 & 0.0000 & -0.0003 & 0.2209 & 0.0000 & -0.1299 & 0.0000 & 0.074 \\
\hline & $\mathrm{G}$ & -0.016 & 0.016 & -0.035 & 0.234 & 0.006 & 0.014 & -0.123 & 0.467 & -0.003 & -0.371 & -0.112 & 0.076 \\
\hline \multirow[t]{2}{*}{ Tillers/plant } & $\mathrm{P}$ & -0.0003 & -0.0009 & 0.0000 & 0.0003 & -0.0207 & 0.0002 & -0.0005 & 0.0533 & 0.0000 & -0.0491 & -0.0004 & -0.018 \\
\hline & G & -0.011 & 0.049 & -0.033 & 0.008 & 0.166 & -0.044 & -0.136 & -0.205 & -0.047 & 0.161 & 0.005 & -0.086 \\
\hline \multirow[t]{2}{*}{ Spike length (cm) } & $\mathrm{P}$ & 0.0006 & 0.0002 & 0.0000 & 0.0001 & -0.0005 & 0.0071 & -0.0008 & -0.0884 & 0.0003 & 0.1056 & 0.0007 & 0.025 \\
\hline & G & 0.028 & -0.002 & 0.008 & -0.024 & 0.054 & -0.134 & -0.004 & -0.222 & 0.027 & 0.257 & 0.039 & 0.027 \\
\hline \multirow[t]{2}{*}{ Peduncle length (cm) } & $\mathrm{P}$ & -0.0016 & -0.0004 & 0.0000 & -0.0010 & -0.0025 & 0.0014 & -0.0040 & 0.0441 & -0.0006 & 0.0365 & -0.0004 & 0.071 \\
\hline & G & -0.043 & 0.079 & 0.027 & 0.117 & 0.091 & -0.002 & -0.247 & -0.362 & -0.019 & 0.924 & -0.160 & $0.404 * *$ \\
\hline \multirow[t]{2}{*}{ Biological yield/plant (g) } & $\mathrm{P}$ & 0.0028 & -0.0014 & 0.0001 & -0.0028 & -0.0009 & -0.0005 & -0.0001 & 1.1792 & -0.0002 & -1.2907 & 0.0007 & $-0.114 *$ \\
\hline & G & 0.062 & 0.040 & -0.048 & 0.086 & -0.027 & 0.024 & 0.071 & 1.270 & -0.027 & -1.652 & 0.006 & $-0.196^{* *}$ \\
\hline \multirow[t]{2}{*}{ Test weight (g) } & $\mathrm{P}$ & 0.0005 & -0.0004 & 0.0000 & -0.0001 & 0.0001 & -0.0003 & -0.0003 & 0.0338 & -0.0075 & 0.0849 & 0.0001 & $0.111^{*}$ \\
\hline & G & -0.002 & 0.032 & -0.001 & 0.005 & 0.053 & 0.024 & -0.032 & 0.237 & -0.145 & 0.129 & -0.022 & $0.278^{* *}$ \\
\hline \multirow[t]{2}{*}{ Harvest index (\%) } & $\mathrm{P}$ & -0.0031 & 0.0015 & -0.0001 & 0.0012 & 0.0006 & 0.0005 & -0.0001 & -0.9370 & -0.0004 & 1.6243 & -0.0010 & $0.687 * *$ \\
\hline & $\mathrm{G}$ & -0.066 & -0.036 & 0.049 & -0.043 & 0.013 & -0.017 & -0.112 & -1.030 & -0.009 & 2.036 & -0.057 & $0.729 * *$ \\
\hline \multirow[t]{2}{*}{ Spikelets/spike } & $\mathrm{P}$ & -0.0013 & -0.0010 & 0.0000 & 0.0000 & -0.0011 & -0.0007 & -0.0003 & -0.1170 & 0.0001 & 0.2289 & -0.0067 & $0.101 *$ \\
\hline & G & -0.111 & 0.008 & 0.086 & 0.231 & -0.007 & 0.047 & -0.351 & -0.064 & -0.028 & 1.025 & -0.113 & $0.724 * *$ \\
\hline
\end{tabular}

*, ** significant at 5 and 1 per cent probability levels, respectively 
Table.3 Estimates of phenotypic and genotypic correlation coefficient between 12 characters under timely sown $\left(\mathrm{E}_{1}\right)$ condition in wheat

\begin{tabular}{|c|c|c|c|c|c|c|c|c|c|c|c|c|c|}
\hline Character & & $\begin{array}{c}\text { Day to } 50 \% \\
\text { flowering }\end{array}$ & $\begin{array}{c}\text { Flag leaf } \\
\text { area }(\mathrm{cm})^{2}\end{array}$ & $\begin{array}{l}\text { Days to } \\
\text { maturity }\end{array}$ & $\begin{array}{c}\text { Plant height } \\
\text { (cm) }\end{array}$ & $\begin{array}{l}\text { Tillers/ } \\
\text { plant }\end{array}$ & $\begin{array}{l}\text { Spike } \\
\text { length } \\
(\mathrm{cm})\end{array}$ & $\begin{array}{c}\text { Peduncle } \\
\text { length }(\mathrm{cm})\end{array}$ & $\begin{array}{l}\text { Biological } \\
\text { yield/ plant } \\
\quad(\mathrm{gm})\end{array}$ & $\begin{array}{c}\text { Test } \\
\text { weight } \\
(\mathrm{gm})\end{array}$ & $\begin{array}{c}\text { Harvest } \\
\text { index }(\%)\end{array}$ & $\begin{array}{c}\text { Spikelets/spik } \\
\text { e }\end{array}$ & $\begin{array}{c}\text { Grain yield/ } \\
\text { plant (gm) }\end{array}$ \\
\hline \multirow{2}{*}{$\begin{array}{c}\text { Days to } 50 \% \\
\text { flowering }\end{array}$} & $\mathbf{P}$ & 1.000 & $0.159^{* *}$ & $0.396^{* *}$ & -0.001 & -0.032 & -0.072 & 0.072 & 0. & -0.022 & $-0.183 * *$ & -0.050 & $-0.149^{* *}$ \\
\hline & G & 1.000 & $0.297^{* *}$ & $0.422^{* *}$ & -0.003 & $0.213^{* *}$ & -0.088 & $0.139 * *$ & $0.213 * *$ & -0.017 & $-0.271 * *$ & -0.063 & $-0.213 * *$ \\
\hline \multirow[t]{2}{*}{ Flag leaf area $\left(\mathrm{cm}^{2}\right)$} & $\mathbf{P}$ & & 1.000 & -0.011 & -0.047 & 0.027 & 0.030 & -0.042 & 0.004 & $-0.116^{*}$ & -0.047 & -0.032 & -0.057 \\
\hline & G & & 1.000 & -0.038 & -0.082 & 0.053 & 0.043 & -0.050 & -0.011 & $-0.184 * *$ & -0.024 & -0.015 & -0.040 \\
\hline \multirow[t]{2}{*}{ Days to maturity } & $\mathbf{P}$ & & & 1.000 & -0.034 & -0.046 & $-0.224 * *$ & $0.191 * *$ & $0.194^{* *}$ & $0.182 * *$ & $-0.271 * *$ & 0.041 & $-0.219 * *$ \\
\hline & G & & & 1.000 & -0.053 & $0.253^{* *}$ & $-0.338 * *$ & $0.327 * *$ & $0.348 * *$ & $0.274 * *$ & $-0.449 * *$ & $0.100^{*}$ & $-0.367 * *$ \\
\hline \multirow[t]{2}{*}{ Plant height (cm) } & $\mathbf{P}$ & & & & 1.000 & -0.033 & -0.026 & 0.067 & -0.054 & $0.115^{*}$ & 0.071 & -0.019 & 0.061 \\
\hline & G & & & & 1.000 & -0.019 & -0.063 & 0.093 & -0.086 & $0.126^{*}$ & $0.102 *$ & 0.008 & 0.083 \\
\hline \multirow[t]{2}{*}{ Tillers/plant } & $\mathbf{P}$ & & & & & 1.000 & 0.011 & 0.076 & 0.008 & $-0.148^{* *}$ & $0.110^{*}$ & 0.051 & $0.154^{* *}$ \\
\hline & G & & & & & 1.000 & 0.028 & $0.366 * *$ & -0.061 & $-0.392 * *$ & $0.352 * *$ & 0.061 & $0.465^{* *}$ \\
\hline \multirow[t]{2}{*}{ Spike length (cm) } & $\mathbf{P}$ & & & & & & 1.000 & -0.046 & 0.008 & $-0.142 * *$ & $0.108^{*}$ & -0.005 & $0.146^{* *}$ \\
\hline & G & & & & & & 1.000 & -0.058 & 0.044 & $-0.181 * *$ & $0.152 * *$ & -0.031 & $0.239 * *$ \\
\hline \multirow[t]{2}{*}{ Peduncle length $(\mathrm{cm})$} & $\mathbf{P}$ & & & & & & & 1.000 & -0.074 & $0.177^{* *}$ & 0.025 & $0.253^{* *}$ & -0.017 \\
\hline & G & & & & & & & 1.000 & $-0.099^{*}$ & $0.205^{* *}$ & 0.031 & $0.454 * *$ & -0.025 \\
\hline \multirow{2}{*}{$\begin{array}{c}\text { Biological yield/plant } \\
\text { (g) }\end{array}$} & $\mathbf{P}$ & & & & & & & & 1.000 & 0.056 & $-0.681^{* *}$ & -0.025 & $-0.183 * *$ \\
\hline & G & & & & & & & & 1.000 & 0.066 & $-0.726^{* *}$ & -0.063 & $-0.264 * *$ \\
\hline \multirow[t]{2}{*}{ Test weight (g) } & $\mathbf{P}$ & & & & & & & & & 1.000 & $-0.204 * *$ & $0.121^{*}$ & $-0.236 * *$ \\
\hline & G & & & & & & & & & 1.000 & $-0.231 * *$ & $0.206^{* *}$ & $-0.279 * *$ \\
\hline \multirow[t]{2}{*}{ Harvest index (\%) } & $\mathbf{P}$ & & & & & & & & & & 1.000 & -0.051 & $0.843 * *$ \\
\hline & G & & & & & & & & & & 1.000 & -0.055 & $0.854 * *$ \\
\hline \multirow[t]{2}{*}{ Spikelets/Spike } & $\mathbf{P}$ & & & & & & & & & & & 1.000 & -0.084 \\
\hline & G & & & & & & & & & & & 1.000 & $-0.116^{*}$ \\
\hline
\end{tabular}

*, ** significant at 5 and 1 per cent probability levels, respectively 
Table.4 Estimates of phenotypic and genotypic correlation coefficient between 12 characters under late sown condition in wheat

\begin{tabular}{|c|c|c|c|c|c|c|c|c|c|c|c|c|c|}
\hline Character & & $\begin{array}{c}\text { Day to } \\
50 \% \\
\text { flowering }\end{array}$ & $\begin{array}{l}\text { Flag leaf } \\
\text { area } \\
(\mathrm{cm})^{2}\end{array}$ & $\begin{array}{l}\text { Days to } \\
\text { maturity }\end{array}$ & $\begin{array}{l}\text { Plant } \\
\text { height } \\
(\mathbf{c m})\end{array}$ & $\begin{array}{c}\text { Tillers/p } \\
\text { lant }\end{array}$ & $\begin{array}{l}\text { Spike } \\
\text { length } \\
(\mathbf{c m})\end{array}$ & $\begin{array}{l}\text { Peduncle } \\
\text { length } \\
\text { (cm) }\end{array}$ & $\begin{array}{l}\text { Biological } \\
\text { yield/ } \\
\text { plant }(\mathrm{gm})\end{array}$ & $\begin{array}{c}\text { Test } \\
\text { weight } \\
(\mathrm{gm})\end{array}$ & $\begin{array}{c}\text { Harvest } \\
\text { index }(\%)\end{array}$ & $\begin{array}{l}\text { Spikelets } \\
\text { /spike }\end{array}$ & $\begin{array}{c}\text { Grain } \\
\text { yield/ plant } \\
\text { (gm) }\end{array}$ \\
\hline \multirow[t]{2}{*}{ Days to $50 \%$ flowering } & $\mathrm{P}$ & 1.000 & $-0.108^{*}$ & -0.066 & $0.128 *$ & 0.020 & -0.037 & 0.097 & $-0.173 * *$ & -0.033 & $0.188 * *$ & 0.079 & 0.081 \\
\hline & $\mathrm{G}$ & 1.000 & $-0.301 * *$ & $-0.151 * *$ & $0.118^{*}$ & 0.083 & $-0.209 * *$ & $0.321 * *$ & $-0.468 * *$ & 0.018 & $0.495 * *$ & $0.828 * *$ & $0.244 * *$ \\
\hline \multirow[t]{2}{*}{ Flag leaf area $\left(\mathrm{cm}^{2}\right)$} & $\mathrm{P}$ & & 1.000 & 0.015 & -0.034 & -0.057 & 0.012 & -0.025 & -0.085 & -0.025 & 0.090 & -0.064 & 0.067 \\
\hline & $\mathrm{G}$ & & 1.000 & $-0.121^{*}$ & $-0.100 *$ & $-0.313^{* *}$ & 0.011 & $-0.500 * *$ & $-0.255 * *$ & $-0.205^{* *}$ & $0.225^{* *}$ & -0.053 & $0.117 *$ \\
\hline \multirow[t]{2}{*}{ Days to maturity } & $\mathrm{P}$ & & & 1.000 & 0.049 & 0.039 & -0.036 & 0.011 & $0.128^{*}$ & -0.023 & $-0.140 * *$ & 0.090 & -0.076 \\
\hline & $\mathrm{G}$ & & & 1.000 & $0.233 * *$ & $0.219 * *$ & -0.052 & $-0.181 * *$ & $0.325 * *$ & 0.005 & $-0.331 * *$ & $-0.577 * *$ & $-0.163 * *$ \\
\hline \multirow[t]{2}{*}{ Plant height (cm) } & $\mathrm{P}$ & & & & 1.000 & -0.022 & -0.004 & 0.065 & $0.187 * *$ & 0.004 & -0.080 & -0.002 & 0.074 \\
\hline & $\mathrm{G}$ & & & & 1.000 & 0.036 & $-0.101^{*}$ & $0.499 * *$ & $0.367 * *$ & 0.023 & $-0.182 * *$ & $0.990 * *$ & 0.076 \\
\hline \multirow[t]{2}{*}{ Tillers/plant } & $\mathrm{P}$ & & & & & 1.000 & 0.026 & $0.123^{*}$ & 0.045 & -0.006 & -0.030 & 0.053 & -0.018 \\
\hline & $\mathrm{G}$ & & & & & 1.000 & $0.329 * *$ & $0.549 * *$ & $-0.162 * *$ & $0.321 * *$ & 0.079 & -0.042 & -0.086 \\
\hline \multirow[t]{2}{*}{ Spike length (cm) } & $\mathrm{P}$ & & & & & & 1.000 & $0.197 * *$ & -0.075 & -0.040 & 0.065 & $-0.103^{*}$ & 0.025 \\
\hline & $\mathrm{G}$ & & & & & & 1.000 & 0.017 & $-0.175^{* *}$ & $-0.182 * *$ & $0.126^{*}$ & $-0.348^{* *}$ & 0.027 \\
\hline \multirow[t]{2}{*}{ Peduncle length $(\mathrm{cm})$} & $\mathrm{P}$ & & & & & & & 1.000 & 0.037 & 0.084 & 0.022 & 0.063 & 0.071 \\
\hline & $\mathrm{G}$ & & & & & & & 1.000 & $-0.285^{* *}$ & $0.131 * *$ & $0.454^{* *}$ & $1.418 * *$ & $0.404 * *$ \\
\hline \multirow{2}{*}{$\begin{array}{l}\text { Biological yield/plant } \\
\text { (g) }\end{array}$} & $\mathrm{P}$ & & & & & & & & 1.000 & 0.029 & $-0.795^{* *}$ & $-0.099 *$ & $-0.114^{*}$ \\
\hline & $\mathrm{G}$ & & & & & & & & 1.000 & $0.186^{* *}$ & $-0.811 * *$ & -0.051 & $-0.196 * *$ \\
\hline \multirow[t]{2}{*}{ Test weight (g) } & $\mathrm{P}$ & & & & & & & & & 1.000 & 0.052 & -0.011 & $0.111^{*}$ \\
\hline & $\mathrm{G}$ & & & & & & & & & 1.000 & 0.063 & $0.194 * *$ & $0.278 * *$ \\
\hline \multirow[t]{2}{*}{ Harvest index (\%) } & $\mathrm{P}$ & & & & & & & & & & 1.000 & $0.141 * *$ & $0.687 * *$ \\
\hline & $\mathrm{G}$ & & & & & & & & & & 1.000 & $0.504 * *$ & $0.729 * *$ \\
\hline \multirow[t]{2}{*}{ Spikelets/Spike } & $\mathrm{P}$ & & & & & & & & & & & 1.000 & $0.101 *$ \\
\hline & G & & & & & & & & & & & 1.000 & $0.724 * *$ \\
\hline
\end{tabular}

*** significant at 5 and 1 per cent probability levels, respectively 
Highest positive indirect effect on grain yield was exerted by days to maturity $(0.141)$ via biological yield per plant followed by days to $50 \%$ flowering (0.092) via biological yield per plant, test weight (0.040) via biological yield per plant, tiller per plant and spike length (0.005) via biological yield per plant and flag leaf area (0.003) via biological yield per plant in $E_{1}$. Besides, $E_{1}$ also have the negative indirect effect on grain yield which was exerted by harvest index $(-0.498)$ via biological yield per plant followed by peduncle length (-0.054), plant height ($0.039)$ and spikelets per spike $(-0.018)$ via biological yield per plant. Positive and highest indirect effect on grain yield was exerted by plant height $(0.220)$ via biological yield per plant followed by days to maturity (0.151), tiller per plant (0.053) peduncle length (0.044), test weight (0.033) and negative indirect effect on grain yield were exerted by harvest index (-0.937) via biological yield per plant followed by days to $50 \%$ flowering ($0.204)$, flag leaf area $(-0.1002)$ and by spike length (-0.088) via biological yield per plant in $E_{2}$ (Table.1 and 2) the indirect effects of remaining characters were too low to be considered as important and the above mentioned characters emerged as most important direct yield contributors on which emphasis should be given during simultaneous selection aimed at improving grain yield in wheat. These characters have also been identified as major direct contributors towards seed yield by Singh, Bhuri and Upadhyay, P.K. (2013), Ayccek and Yldrm (2006), Sherif et al., (2005), Payal et al., (2007), Dharmendra and Singh (2010), Tripathi et al., (2011), El-Mohsen et al., (2012) and Bhutto et al., (2015).

\section{Correlation coefficient}

Correlation study of twelve traits revealed that besides grain yield traits are also correlated with each other. Thus, selection practiced for improving these traits individually or simultaneously would bring improvement in other due to correlated response. This suggested that selection would be quite efficient in improving yield and yield components in wheat (Table. 3 and 4).

Grain yield per plant exhibited highly positive phenotypic correlation with harvest index (0.843), tiller per plant (0.154) and spike length (0.146) in $E_{1}$ and negative correlation for the $\mathrm{E}_{1}$ with test weight (-0.236), days to maturity $(-0.219)$, biological yield per plant (0.183 ) and days to $50 \%$ flowering (-0.149), while grain yield per plant exhibited highly positive phenotypic correlation with harvest index (0.687), test weight (0.111) and spikelet per spike $(0.101)$ in $E_{2}$. In $E_{2}$ also showed negative phenotypic correlation with biological yield per plant (-0.114).Days to maturity showed positive correlation with days to $50 \%$ flowering (0.396) in $\mathrm{E}_{1}$ and remaining traits were non-significant in $\mathrm{E}_{1}$ as well as in $\mathrm{E}_{2}$.

Flag leaf area showed highly positive correlation with days to $50 \%$ flowering $(0.159)$ in $E_{1}$. whereas for $E_{2}$ showed negative association with days to $50 \%$ flowering (0.108), whereas plant height showed nonsignificant correlation with in $\mathrm{E}_{1}$, whereas, in $\mathrm{E}_{2}$ days to $50 \%$ flowering (0.128), and rest of the characters either very less or nonsignificant, whereas tillers per plant showed non-significant correlation for all the characters in $E_{1}$ as well as for $E_{2}$ and spike length showed negative association with days to maturity (-0.224) and rest of all the characters were non-significant in $\mathrm{E}_{1}$, while $\mathrm{E}_{2}$ possessed non-significant association.

Peduncle length was positively correlated with days to maturity (0.191), while remaining traits were of non-significant in $\mathrm{E}_{1}$, On the other hand, peduncle length showed positively correlation with spike length 
(0.197) and tiller per plant (0.123);remaining traits were non-significant in $\mathrm{E}_{2}$.

Biological yield per plant showed positively association with days to maturity (0.194) and days to $50 \%$ flowering (0.127) and remaining traits were non-significant in $\mathrm{E}_{1}$. while Biological yield per plant showed positive association with plant height (0.187), days to maturity (0.128) and negative correlated with days to $50 \%$ flowering $(-0.173)$ in $\mathrm{E}_{2}$.

Test weight showed positively associated with days to maturity (0.182), penduncle length (0.177), plant height (0.115) and negatively correlated with tiller per plant (-0.148), spike length (-0.142), flag leaf area (-0.116) in $\mathrm{E}_{2}$ it showed non-significant correlation with all characters, while harvest index showed highly positive significant correlation with tillers per plant (0.110), spike length (0.108) and negatively correlated with biological yield per plant $(-0.681)$, days to maturity $(-0.271)$, test weight $(-0.204)$ and days to maturity $(-0.183)$ in $E_{1}$. While in $E_{2}$ showed positive correlation with days to $50 \%$ flowering (0.188), $\mathrm{E}_{2}$ negative correlation with biological yield per plant $(-0.795)$, days to maturity $(-0.140)$.

Spikelets per spikes content showed highly significant correlation with peduncle length (0.253), test weight (0.121) in $E_{1}$. While in $E_{2}$ it was positively correlated with harvest index $(0.141)$ and negatively associated with spike length (-0.103), biological yield (-0.099) and same study have also been reported by Prasad et al., (2006), Payal et al., (2007), Yousaf et al., (2008), Nagireddy and Jyothula (2009), El-Mohsen et al., (2012), Bhutto et al., (2015).

In conclusions the present study shows that Grain yield per plant exhibited highly positive phenotypic correlation with harvest index, test weight and spikelet per spike in $\mathrm{E}_{2}$ and harvest index, tiller per plant, spike length showed highly positive phenotypic correlation with in $\mathrm{E}_{1}$ for grain yield. The highest positive direct effect on grain yield per plant were exerted by harvest index, plant height, penduncle length, whereas $E_{1}$ showed direct positive effect on grain yield per plant by harvest index, biological yield and flag leaf area. Hence, These characters should be given due consideration during selection for yield improvement of wheat.

\section{References}

Anonymous (2019). Area production and yield of India \& state Agriculture Statistics at a Glance. Government of India, Ministry of Agriculture \& Farmers Welfare Department of Agriculture, co-operation \& Farmer Welfare, Directorate of Economics and Statistics, Pp-71-79.

Esmail, R.M. (2001). Correlation and pathcoefficient analysis of some quantitative traits with grain yield in bread wheat (Triticum aestivum L.). Bulletin of the National Centre, Cairo., 26(3): 395-408.

Lohithaswa, H. C., Desai, S. A., Hanchinal, R. R., Patil, B. N., Math, K. K., Kalappanavar, I. K., Bandivadder, T. T. and Chandrashekhara, C. P. (2013). Combining ability in tetraploid wheat for yield, yield attributing traits, quality and rust resistance over environments. Karnataka J. Agric. Sci., 26(2): 190-193.

Meena, H. S., Kumar, Dinesh and Prasad, S. R. (2014). Genetic variability and character association in bread wheat (Triticum aestivum L.).Indian Journal of Agricultural Sciences 84(4):487491.

Mishra, Upasna., Sharma, A.K. and Chauhan, Shailja. (2019). Genetic variability, heritability and genetic advance in bread wheat (Triticum aestivum L.). 
Int.J.Curr.Microbiol.App.Sci. $\quad$ 8(7): 2311-2315

Molla, A. and Thomas, L. (2009).Genetic analysis of wheat varieties for yield and its components. Ann. Bio., 25(1): 31-34.

Adhikari, Anil, Amir, Ibrahim M.H., Rudd, Jackie, C., Baenziger, P. Stephen and Sarazin, Jean-Benoit (2019). Estimation of heterosis and combining abilities of U.S. winter wheat germplasm for hybrid development. Crop Sci., 60(2): 788-803

Asif, M., Mujahid, M.Y., Kisana, N.S., Mustafa, S.Z. and Ahmad, I. (2004). Heritability, genetic variability and path-coefficients of some traits in spring wheat. Sarhad $J$. of Agril.Pakistan., 20(1): 87-91.

Ayccek, M. and Yldrm, T. (2006).Path coefficient analysis of yield and yield components in bread wheat (T. aestivum L.). Pak. J. of Botany.,38(2): $417-424$.

Bergale, S., Mridula Billore, Holkar, A. S. Ruwali, K. N. and Prasad, V. S. (2002). Pattern of variability, character association and path analysis inwheat (Triticum aestivum L.). Agril.Sci. Digest., 22(4): 258-260.

Bhutto, A., Rajpar, A., Kalhoro, S., Ali, A., Kalhoro, F., Ahmed, M., Raza, S. and Kalhoro, N. (2016) Correlation and Regression Analysis for Yield Traits in Wheat (Triticum aestivum L.) Genotypes. Natural Science, 8: 96104.

Dhadhal, B. A., Dobariya, K. L., Ponkia, H. P. and Jivani, L. L. (2008). Gene action and combining ability over environments for grain yield and its attributes in bread wheat (Triticum aestivum L.). I. J. Agric. Sci., 4(1):6672.

Adhikari, Anil, Amir, Ibrahim M.H., Rudd, Jackie, C., Baenziger, P. Stephen and
Sarazin, Jean-Benoit (2019). Estimation of heterosis and combining abilities of U.S. winter wheat germplasm for hybrid development. Crop Sci., 60(2): 788-803

Asif, M., Mujahid, M.Y., Kisana, N.S., Mustafa, S.Z. and Ahmad, I. (2004). Heritability, genetic variability and path-coefficients of some traits in spring wheat. Sarhad J. Agril. Pakistan., 20(1): 87-91.

Ayccek, M. and Yldrm, T. (2006).Path coefficient analysis of yield and yield components in bread wheat ( $T$. aestivum L.). Pak. J. of Botany., 38(2): $417-424$

Bergale, S., MridulaBillore, Holkar, A. S. Ruwali, K. N. and Prasad, V. S. (2002). Pattern of variability, character association and path analysis in wheat (Triticum aestivum L.). Agril. Sci. Digest., 22(4): 258-260.

Bhutto, A., Rajpar, A., Kalhoro, S., Ali, A., Kalhoro, F., Ahmed, M., Raza, S. and Kalhoro, N. (2016) Correlation and regression analysis for yield traits in wheat (Triticum aestivum L.) Genotypes. Natural Science, 8: 96104.

Dhadhal, B. A., Dobariya, K. L., Ponkia, H. P. and Jivani, L. L. (2008). Gene action and combining ability over environments for grain yield and its attributes in bread wheat (Triticum aestivum L.). I. J. Agric. Sci., 4(1):6672.

Pancholi, S. R., Sharma, S. N., Sharma, Y. and Maloo, S. R. (2012).Combining ability computation from diallel crosses comprising ten bread wheat cultivars. Crop Res., 43(1/2/3): 131141.

Panwar, B. S. and Singh, D. (2000). Genetic variability and correlation studies in wheat. Indian J. Pl. Genet. Resources, 13(3): 286-289. 
Payal, Saxena.,Rawat, R. S., Verma, J. S. and Meena, B. K. (2007).Variability and association analysis for yield and quality traits in wheat. Pant. J. of Res., 5(2): 85-92.

Prasad, J., Kerketta, V., Prasad, K. D. and Verma, A. K. (2006).Study of genetic parameters under different environment conditions in wheat (Triticum aestivum L.). J. Res. Birsa Agril. Uni., 18(1): 135-140. 183

Rangare, N. R., Krupakar, A., Kumar, A. and Singh, S. (2010). Character association and component analysis in wheat (Triticum aestivum L.). Electronic J. Pl. Breed., 1(3):231-238.

Rathod, S.T., Pole, S.P. and Gawande, S.M. (2019). Correlation and path analysis for quality and yield contributing traits in wheat (Triticum aestivum L.). Int.J.Curr.Microbiol.App.Sci.,8(6): $456-461$

Rawlings, J. O. and Cokerham, C. C. (1962a). Triallele analysis. Crop Sci., 228-231.

Rawlings, J. O. and Cokerham, C. C. (1962b). Analysis of double cross hybrid populations. Biometrics, 18: 229-244.

Redden, R. J. and Jensen, N. F. (1974).Mass selection and mating system in cereals. Crop Sci., 14: 345-350.

Riaz-ud-Din Subhani, G. M. Naeem, Ahmad. Makhdoom, Hussain and Aziz-urRehman (2010). Effect of temperature on development and grain formation in spring wheat. Pak. J. of Botany., 42(2): 899-906.

Seleem, S. A. and El- Sawi, S. A. (2006). Line $\mathrm{x}$ tester analysis for grain yield and its components in bread wheat.Minufiya J. Agric. Res.,31(1): 75-87.

Shabbir, G., Kiran, T., Akram, Z., Tabassum, M. I. and Shah, K. N. (2012).Genetics of some biometric traits in bread wheat (Triticum aestivum L.). J. Agric. Res., 50(4): 457-468.
Shahnaz, M., Ansari, B. A. and Shabana, M. (2005).Evaluation of bread wheat progenies derived from $4 \times 4$ parent diallel cross of spring wheat ( $T$. aestivum L.). Indus J. Pl. Sci., 4(2): 142-145.

Sharma, S. and Chaudhary, H. K. (2009). Combining ability and gene action studies for yield contributing traits in crosses involving winter and spring wheat genotypes. Acta-Agronomica Hungarica, 57(4): 417-423.

Sherif, H. S., Hosary. E. L., Behit, M. M., Moustafa, M. A. and Maghraby,

M. A. (2005).Correlation and Path coefficient analysis of yield characters in bread wheat (T.aestivum L.). Ann. Agril. Sci. Moshtohor., 43(4): 1677-1687.

Siddique, M., Ali, S., Malik, M. F. A. and Awan, S. I. (2004). Combining ability estimates for yield and yield components in spring wheat. Sarhad J. Agric., 20(4): 485-487.

Singh, B. N., Soni, S. K., Srivastava, A. and Yadav, V. K. (2012). Analysis of yield components and their association for selection ofparent to architecture model plant type in bread wheat (Triticum aestivum L.) to saline soil. Environ. and Ecology., 30(1): 106109.

Singh, Bhuri and Upadhyay, P.K. (2013). Genetic variability, correlation and path analysis in wheat (Triticum aestivum L.). Internat. J. Plant Sci., 8 (2): 230-235.

Sprague, G. F. and Tatum, L. A. (1942).General vs. specific combining ability in single crosses of corn.J. Amer. Soc. Agron., 34: 923-932.

Tripathi, S. N., Marker, S., Pandey, P., Jaiswal, K. K. and Tiwari, D. K. (2011). Relationship between some morphological and physiological traits with grain yield in bread wheat (Triticum aestivum L. em. Thell.). 
Trends in App. Sci. Res., 6(9):10371045.

Ullah, S. U., Khan, A. S., Raza, A. and Sadique, S. (2009). Gene action analysis of yield and yield related traits in spring wheat (Triticum aestivum L.). I. J. Agric. Bio., 12(1): 125-128.

Vanpariya, L. G., Chovatia, V. P. and Mehta, D. R. (2006a). Combining ability studies in bread wheat (Triticum aestivum L.).National J. Pl.Improv., 8(2): 132-137.

Verma, P. N., Singh, B. N. and Yadav, R. K. (2013). Genetic variability and divergence analysis of yield and its contributing traits under sodic soil condition in wheat (T. aestivum L.). I. J. Agril. Sci., 3(2): 395-399.

Vishwa Mohan TNN (2015). Food grain production during 2014-15 crop year declines by $13.92 \mathrm{mt}$. The Times Of India, Pp. 1-3.

Yadav S K, Singh, A K, Baghel, S S, Jarmanand, M, Singh, A K. 2014. Assessment of Genetic variability and Diversity for Yield and its Contributing Traits among CIMMYT Based Wheat Germplasm. Journal of Wheat Research 6(1):154-159.
Yousaf Ali., Atta, B. M., Akhter, J., Monneveux, P., and Lateef, Z. (2008). Genetic variability, association and diversity studies in wheat (Triticum aestivum L.) germplasm. Pak. J. Bot., 40(5): 2087-2097.

Zahid, A., Ajmal, S. U., Khan, K. S., Qureshi, R. and Zubair, M. (2011). Combining ability estimates of some yield and quality related traits in spring wheat (Triticum aestivum L.). Pak. J. Bot., 43(1): 221-231.

Zahid, A., Ajmal, S. U., Munir, M. and Shabir, G. (2008). Genetic determination of yield related attributes in bread wheat. Sarhad J. Agric., 24(3): 431-438.

Zalewski, D. (2001).Estimation of general and specific combining ability of quantitative traits of winter wheat. Biuletyn Instytutu Hodowli-iAklimatyzacji Roslin, 216(2):267-272.

Zine, E., Fellahi, A., Hannachi, A., Bouzerzour, H. and Boutekrabt, A. (2013).Line $\times$ tester mating design analysis for grain yield and yield related traits in bread wheat (Triticum aestivum L.). I. J. Agron., Article ID 201851, 9: 46-49.

\section{How to cite this article:}

Singh, K. P., Vinod Singh, Gaurav Sharma, M. P. Chauhan, Tejasvi Singh and Yadav, R. D. S. 2020. Correlation and Path Coefficient Analysis in Wheat (Triticum aestivum L. em. Thell). Int.J.Curr.Microbiol.App.Sci. 9(11): 1570-1581. doi: https://doi.org/10.20546/ijcmas.2020.911.186 